\title{
Universidad y protesta social: una reflexión desde Chile
}

\author{
José-Joaquín Brunner, Francisco Ganga-Contreras y Julio Labraña-Vargas
}

\section{RESUMEN}

Desde octubre de 2019, Chile experimentó una intensa protesta social, a la cual luego se sobreponen los efectos de la pandemia del Covid-19. Este ensayo indaga en las causas de la protesta y el rol que desempeñan las universidades en tiempos de crisis. Para esto recurre a conceptos de la sociología de la educación superior y utiliza datos secundarios provenientes del debate público, la prensa, informes de agencias gubernamentales y observaciones recogidas por estudios de casos. Concluye que la protesta fue provocada por una brecha entre expectativas de participación en los beneficios de la modernización capitalista, movilizadas por la masificación de la educación superior, y las satisfacciones generadas por el Estado y la sociedad. En este cuadro, las universidades se han debatido entre abstraerse de las luchas sociales, plegarse a ellas o contribuir a superar la crisis desde su propia racionalidad institucional.

Palabras clave: Chile, educación superior, papel de la universidad, clase media, conflictos sociales.

José-Joaquín Brunner

Chileno, Doctor en Sociología por la Universidad de Leiden, Holanda. Investigador del Centro de Políticas Comparadas de Educación, Universidad Diego Portales; Profesor Titular de la Universidad Diego Portales, Chile, y Director de la Cátedra unEsco de Políticas Comparadas de Educación Superior. Temas de investigación: economía política y gobernanza de la educación superior, análisis de sistemas, políticas comparadas.
Francisco Ganga-Contreras

franciscoganga@academicos.uta.cl

Chileno, Doctor en Gestión Estratégica y Negocios Internacionales, Universidad de Sevilla-España. Postdoctorado en Ciencias Humanas, Universidad del Zulia-Venezuela. Consultor Internacional, Profesor Titular del Departamento de Educación de la Facultad de Educación y Humanidades de la Universidad de Tarapacá-Chile. ORCID: https://orcid.org/0000-0001-9325-6459. Web personal: franciscoganga.cl. Temas de investigación: administración de organizaciones y gobernanza universitaria, gobernanza de entidades educativas.

\section{Julio Labraña-Vargas}

Chileno, Doctor en Filosofía por la Universidad Witten/Herdecke, Alemania. Investigador asociado de la Universidad Diego Portales, Chile; Coordinador Ejecutivo de la Cátedra unEsco de Políticas Comparadas de Educación Superior e Investigador del Núcleo de Estudios Sistémicos Transdisciplinarios. Temas de investigación: sociología de la educación superior, historia de la educación, el vínculo entre cabios estructurales y semántica. 


\section{Universidade e protesto social: uma reflexão do Chile}

\section{RESUMO}

Desde outubro de 2019, Chile experimentou um intenso protesto social, ao qual logo se sobrepõem os efeitos da pandemia do Covid-19. Este ensaio indaga sobre as causas do protesto e o papel que desempenham as universidades em tempos de crise. Para isto recorre a conceitos da sociologia da educação superior e utiliza dados secundários provenientes do debate público, a imprensa, informes de agências governamentais e observações recolhidas por estudos de casos. Conclui que o protesto foi provocado por uma brecha entre expectativas de participação nos benefícios da modernização capitalista, mobilizadas pela massificação da educação superior, e as satisfações geradas pelo Estado e a sociedade. Neste quadro, as universidades têm debatido entre abstrair-se das lutas sociais, juntar-se a elas ou contribuir a superar a crise desde sua própria racionalidade institucional.

Palavras chave: Chile, educação superior, papel da universidade, classe média, conflitos sociais.

\section{University and social protest: a reflection from Chile}

\section{ABSTRACT}

Since October 2019, Chile experienced an intense social protest, which was later overcome by the effects of the Covid-19 pandemic. This essay explores the causes of the protest and the role of universities in times of crisis. It draws on concepts from the sociology of higher education and makes use of secondary data from public debate, the press, government agency reports, and from case study observations as well. The author concludes that the protest was caused by a gap between the expectations of participation in the benefits of capitalist modernization, mobilized by the massification of higher education, and the satisfactions generated by the state and the society as a whole. In this context, the universities have debated between withdrawing from the social struggles, yielding to them or contributing to overcoming the crisis from their own institutional rationality.

Key words: Chile, higher education, role of the university, middle class, social conflicts. 


\section{Introducción}

Probablemente 2019 y lo que va corrido de 2020 entrarán en la historia de Chile como un tiempo de protesta social masiva que remeció al país, sin dejar indiferente a nadie y generando disímiles emociones: desde un profundo anhelo de cambio - "Chile despertó", decía la consigna - hasta sentimientos de angustia e impotencia frente a saqueos y variadas manifestaciones de indisciplina social; rabia ante el vandalismo destructor y una justa indignación con los abusos de la represión policial. ${ }^{1}$ A esto se suman luego los efectos de la pandemia del Covid-19 que paralizaron el normal transcurso de la vida social, imponiendo nuevos patrones de conducta individual y social (Castiglion, 2020).

Las universidades, a su vez, jugaron un rol preponderante por el activo papel de los graduados y estudiantes en las manifestaciones de protesta, coherente con una tradición de alineamiento con el cambio social y político. Al mismo tiempo, de cara a la sociedad, ofrecieron un punto de encuentro y reflexión sobre los motivos de la protesta, la justicia de las demandas y el carácter de la violencia y su represión. Internamente pusieron en discusión sus relaciones con la sociedad y se interrogaron respecto a cuál debía ser su contribución para la superación de los problemas relevados por la protesta. Y, más adelante, también por la crisis sanitaria.

El presente ensayo analiza el vínculo entre universidad y protesta social masiva en Chile, partiendo del supuesto de que la rápida expansión del acceso a la educación superior (ES) y la consiguiente inflación de diplomas profesionales y técnicos proporcionan una clave sociológica para la comprensión del estallido social de octubre. En efecto, el dramático crecimiento de los graduados de la enseñanza superior explica el abrupto surgimiento de nuevos estratos de clase baja no-pobre y de estratos vulnerables de clase media, resultado de las transformaciones modernizadoras experimentadas por la sociedad chilena durante los últimos 30 años. Como argumentaremos, esta transformación incide además en el carácter de las demandas movilizadas durante la protesta, cuyo eje es un mayor acceso a los beneficios del bienestar capitalista y el reconocimiento de derechos sociales propios de un Estado benefactor que el sistema chileno satisface sólo parcial y desigualmente.

\section{Breve caracterización de la educación superior chilena}

La ES chilena se organiza sobre la base de un sistema de economía política mixta, con provisión predominantemente privada y financiamiento compartido entre el Estado y las familias (Brunner y Ganga, 2016). Dispone de acceso casi universal con una tasa bruta de participación de un $88 \%$, una de las más altas del mundo (UIS, 2019). La matrícula actual es de 1260000 de estudiantes, repartida entre 151 instituciones en diferentes niveles: universitario (61 instituciones), profesional (43 instituciones) y técnico (47 instituciones) (CNED, 2020). Según su base jurídica, las instituciones son de tres tipos: estatales, privadas subvencionadas (con subsidio fiscal directo) y privadas independientes (sin subsidio fiscal directo) que en la nomenclatura de las estadísticas internacionales corresponden a instituciones públicas, privadas dependientes y privadas independientes (UNESCO, 2020).

Los docentes son alrededor de 90000 distribuidos en aproximadamente 400 sedes, que ofrecen más de 10000 programas y gradúan alrededor de 240000 profesionales y técnicos anualmente (CNED, 2020). En 2019, el número de publicaciones indexadas en las bases WoS fue de 12627 artículos, generados por cerca de 10500 autores (CONICyT, 2020).

En cuanto a jerarquía reputacional, las instituciones de ES chilenas se ordenan de acuerdo con diversos

\footnotetext{
${ }^{1}$ Sobre este evento hay ya una variada literatura proveniente de las ciencias sociales (Araujo, 2020; González y Le Foulon, 2020; Peña, 2020; Ruiz, 2020a; Somma, Bargsted y Dissi, 2020; Tironi, 2020). Para un recuento reflexivo de los sucesos de octubre, véase Martuccelli (2020).
} 
criterios, entre los cuales inciden especialmente su naturaleza jurídica, clase de institución (universidad, instituto profesional o centro de formación técnica), año de creación (antigüedad), complejidad organizacional (densidad de conocimiento, investigación y posgrados), nivel de prestigio de su personal académico, grado de selectividad de los estudiantes (si la institución es de élite nacional, semi-élite nacional o regional, de reclutamiento mesocrático - grandes, medianas o pequeñas-, o de acceso libre y sin selección académica) y según el lugar ocupado en rankings nacionales e internacionales.

Un rasgo distintivo del sistema chileno de ES en el contexto internacional comparado es que un $85 \%$ de la matrícula total se registra en instituciones privadas. Asimismo, el hecho de que hasta 2016 todos los estudiantes pagaban un arancel, cualquiera fuese la naturaleza jurídica de su institución, aunque una fracción significativa obtenía algún tipo de beca que cubría parcial o íntegramente el costo de los estudios. Hasta ese momento, cada institución determinaba el precio de sus aranceles, los cuales se consideran elevados (de acuerdo con su valor declarado o de etiqueta) dentro del contexto internacional (OECD, 2019: 314-331). Con todo, existen esquemas de crédito estudiantil a los cuales pueden recurrir los alumnos de menores recursos, los cuales con el tiempo alcanzaron amplia cobertura, generando una onerosa deuda para numerosos graduados, en especial aquellos que no conseguían completar sus estudios (González López, 2018; Disi Pavlic, 2018).

Desde hace un tiempo, el movimiento estudiantil (de la enseñanza secundaria y superior) venía reclamando con fuerza contra la privatización y mercantilización de la ES chilena. A partir de 2006 comenzó una ola de protestas que exigía el fin del lucro y una ES pública gratuita y de calidad (GuzmánConcha, 2012; Disi Pavlic, 2020; Labraña, 2018). En respuesta, la administración Bachelet (2014 a 2018) impulsó una amplia reforma que consagró, a partir de 2015, la gratuidad de los estudios superiores para los jóvenes universitarios provenientes del $60 \%$ de la población de menores recursos, o del $70 \%$ en el caso de los estudiantes de institutos profesionales y centros de formación técnica (Brunner y Ganga, 2018; Brunner y Labraña, 2018).

A pesar de estos cambios, el financiamiento de la ES chilena mantiene hasta hoy un carácter mixto, con participación de recursos provenientes de fuentes estales y privadas (hogares) en proporciones similares. A su vez, el 73\% de los recursos estatales son asignados bajo la modalidad de subsidios a la demanda (estudiantes) para cubrir el costo de la gratuidad (34\%) y de los esquemas de créditos estudiantiles y becas (39\%), mientras que el $27 \%$ restante se destina directamente a las universidades estales (16) y privadas subvencionadas (9), en mayor parte de manera competitiva o vinculado a desempeño y resultados (GGR, 2018a, 2018b). En breve, tal era la fisonomía de la ES chilena al momento de producirse el estallido social.

\section{Protesta social y estado de excepción}

En octubre de 2019 se desencadena en Chile una protesta social masiva (Martuccelli, 2020), cuyo origen puntual estuvo relacionado con la evasión (no pago) por parte de un grupo de estudiantes secundarios del pasaje del Metro (tren subterráneo) de la ciudad de Santiago, como reacción - se proclamó - frente al alza de precios anunciada días antes por la empresa pública que administra este medio de transporte y que había entrado en vigor el 6 de octubre de 2019. Pronto dicha evasión dio paso a la acción violenta de jóvenes encapuchados que el 18 de octubre tomaron por asalto las estaciones del Metro, dañando 77 de las 136 existentes; 6 fueron totalmente incendiadas, 14 fueron quemadas de manera parcial y 57 sufrieron múltiples daños (Metro de Santiago, 2019).

Ese mismo día el gobierno decretó un estado de emergencia (uno de los tipos de estado de excepción constitucional), mandatando a las Fuerzas Armadas 
para que, junto a la policía de carabineros, se hicieran cargo del orden en las calles. Entre sus considerandos el decreto establece que en esa fecha:

han tenido lugar en diversos sectores de la Región Metropolitana múltiples atentados contra la propiedad pública y privada, especialmente contra medios de transporte público de pasajeros, lo que se ha materializado en la destrucción de buses y la total paralización de la red del Metro de Santiago, incluyéndose respecto de este último servicio la quema y destrucción de sus bienes e instalaciones en diversas estaciones, además de la quema, saqueo y destrucción de edificios y locales comerciales (Ministerio del Interior y Seguridad Pública (MIPS), 2019).

De igual manera, señala:

se han desarrollado durante la jornada numerosas barricadas que han impedido la adecuada circulación de vehículos y personas a través de la ciudad [provocándose] una grave alteración del orden público, frente a la cual el Estado debe actuar con todos los medios disponibles, con el objeto de asegurar la integridad, seguridad y tranquilidad de sus habitantes, requiriéndose de medidas extraordinarias orientadas a la protección de las personas y sus bienes". En conformidad con estas consideraciones, el gobierno procedió a declarar el estado de excepción constitucional por un plazo de 15 días

La medida, cuestionada por un importante sector de la sociedad, se extendió por ocho días junto con un toque de queda que obligaba a la población a permanecer en sus hogares durante las horas de la noche. A pesar de las drásticas disposiciones, la protesta civil no amainó; al contrario, se extendió a todo el territorio nacional. A su amparo crecieron también las acciones violentas, multiplicándose las barricadas en las calles, los saqueos de lugares comerciales, los ataques a comisarías y a otros espacios públicos y privados. Por momentos la situación escapó al control del Estado y éste pareció superado, creándose la percepción de una crisis de gobernabilidad.

El 25 de octubre, una convocatoria de los protestantes a marchar pacíficamente en las ciudades de todo el país a través de las redes sociales, reunió en el centro de Santiago a cerca de 1.2 millones de personas que concurrieron a manifestar su descontento con las condiciones de su existencia, su rechazo a la política de los partidos y el gobierno, su irritación frente a los abusos de poder y la corrupción, su desconfianza frente a las instituciones establecidas y a las élites en general, junto con numerosas y variadas demandas propias de las esferas pública y privada. Los manifestantes respondían así a la consigna de un nuevo despertar y la radicalidad de sus demandas expresaba el repudio a la normalidad establecida.

Este sentimiento recorrió a la capital, pero se extendió rápidamente además al resto del territorio nacional, incluyendo a las ciudades grandes, medianas y a un importante número de pequeñas poblados. En vez de símbolos partidistas, las calles se llenaron de banderas, gritos y pancartas que daban cuenta de la diversidad y transversalidad de la protesta, incluyendo pueblos originarios, barras de clubes de fútbol, asociaciones LGTB, expresiones de los movimientos de mujeres, estudiantes, trabajadores, profesionales y a familias enteras, con sus miembros de todas las edades, generándose a ratos un clima de un verdadero carnaval, donde la normalidad se suspende y las relaciones de poder se trastocan, dando paso a las energías de las masas emancipadas de cualquier control. ${ }^{2}$

En los márgenes de la protesta, grupos violentos enfrentaban a la policía, destruían propiedad pública

${ }_{2}^{2}$ Para un análisis de elementos carnavalescos en la protesta a propósito del movimiento estudiantil chileno del 2011, véase Guerra (2015). 
y privada, echaban abajo señalética y monumentos, prendían fuego a iglesias y hoteles, saqueaban centros comerciales e incluso hospitales, armaban barricadas y sembraban terror en el corazón de la ciudad y en los barrios periféricos, mientras las fuerzas encargadas del orden y la seguridad se hallaban desbordadas y reaccionaban con un uso desmedido de la fuerza y los medios represivos, causando centenares de víctimas de violaciones de derechos humanos, lo que a su turno incentivaba la protesta, alimentaba la violencia y corroía la legitimidad de la acción policial. ${ }^{3}$ Así, una espiral fatídica de ingobernabilidad y desorden creó un cuadro de anomia en la vida cotidiana y dejó al descubierto la inefectividad de la institucionalidad democrática. ${ }^{4}$

Cuando el desborde del orden público y la crisis de gobernabilidad amenazaban con apoderarse de la sociedad y en la población crecía la exasperación, el miedo y la incertidumbre, luego de casi 30 días de manifestaciones y caos en las calles, los partidos políticos de gobierno y oposición suscribieron con urgencia un pacto de refundación institucional como perspectiva de salida de la crisis. Éste proclamaba la voluntad transversal de las fuerzas políticas representadas en el Congreso de abrir paso a un proceso que llevase a una nueva Constitución Política del Estado chileno, conjuntamente con el diseño de un plan para recuperar el orden frente a la violencia y de elaborar un plan de medidas sociales para responder a las demandas de la sociedad movilizada.

De esta manera, el 15 de noviembre de 2019, casi la totalidad de las fuerzas políticas representadas en el Parlamento suscribieron el "Acuerdo por la Paz Social y nueva Constitución”, en el que se comprometen a convocar a un plebiscito para consultar a la ciudadanía si respalda la creación de una convención encargada de elaborar una nueva carta fundamental cuyos contenidos deberán aprobarse por 2/3 de sus miembros y ser sometidos posteriormente a ratificación mediante un segundo plebiscito popular. Concurrentemente se pusieron en marcha una serie de medidas para recuperar gradualmente el orden en las calles y se abrió una fase de discusión y aprobación de sucesivas medidas con el propósito de atender demandas de carácter social de la población. Con esto se ponía en marcha una vasta

\footnotetext{
${ }^{3}$ Para una interpretación político-social y estética a la violencia durante la protesta, véase Stange et al. (2019).

${ }^{4}$ No existe un balance consolidado de los costos humanos y económicos de la protesta social y la represión policial frente a la violación de los derechos humanos. Por el lado de los derechos humanos, La Oficina de la Alta Comisionada de las Naciones Unidas para los Derechos humanos reportó el 13 de diciembre de 2019 la existencia de numerosos atropellos y maltratos a los derechos de las personas. Señala que más de 28000 personas fueron detenidas entre el 18 de octubre y el 6 de diciembre, aunque la mayoría de ellas fueron puestas en libertad. Documenta 113 casos específicos de tortura y malos tratos, y 24 casos de violencia sexual contra mujeres, hombres, y niños y niñas adolescentes. Asimismo, informa que el Ministerio Público indicó que existen investigaciones en curso relacionadas con 26 muertes ocurridas en el contexto de las protestas. Afirma que ha habido un uso innecesario y desproporcionado de armas menos letales, en particular escopetas antidisturbios, durante manifestaciones pacíficas o fuera del contexto de enfrentamientos violentos entre manifestantes y fuerzas de seguridad. Además, asegura que el número alarmantemente alto de personas con heridas oculares o faciales, alrededor de 350, demuestra que "hay razones fundadas para creer que se usaron armas menos letales de manera inadecuada e indiscriminada (Noticias ONU, 13 de diciembre de 2019). Por el lado de los costos económicos, después de 30 días de iniciadas la protesta y la violencia, algunas cifras (estimadas) muestran lo siguiente: pérdida de cerca de 3000 millones de dólares, que corresponde al 1.08\% del Producto Interno Bruto (PIB); daño en infraestructuras de alrededor 4500 millones de dólares; caída de un $10 \%$ en las ventas del comercio, de $36 \%$ en las industrias del turismo y el entretenimiento con un $46 \%$ de las empresas del sector afectadas; recaudación del Impuesto al Valor Agregado (IVA) que durante la crisis cayó en un 25\%; según la Encuesta de Expectativas Económicas (EEE), sondeo mensual realizado por un grupo de expertos, señala que el PIB cerrará en 2019 con un crecimiento del $1.9 \%$ - frente al 2.5\% proyectado antes de la crisis—y que se expandirá en 2020 a una tasa del 2.3\%, lejos del $3.5 \%$ de la encuesta anterior; la Cámara de Comercio de Santiago calcula una pérdida de 100000 puestos de trabajo, especialmente en el sector comercio, mientras que la Multigremial Nacional de Emprendedores estima que la pérdida en las pequeñas y medianas empresas habría sido hasta ese momento de 50000 empleos; el precio del dólar dio, por su parte, un salto igualmente brusco, el mayor desde 2002, lo cual motivó a que el Banco Central anunciase una inyección de 4000 millones de dólares para contener la tendencia alcista; también el Índice de Precio Selectivo de Acciones (IPSA), principal índice bursátil de Chile, se vio afectado por la apreciación del dólar y acumulaba una caída del 12.5\% desde el inicio de la crisis, lo que se traduce en una pérdida de 27884 millones de dólares en capitalización bursátil (El Economista América, 14 de noviembre de 2019).
} 
operación destinada a superar la crisis de gobernabilidad, definir un nuevo orden constitucional, satisfacer legítimas demandas sociales y recuperar la normalidad de la vida cotidiana.

\section{Percepciones de la protesta social}

Un sondeo de opinión realizado entre los protestantes en Santiago de Chile constató que tanto la protesta social como los actos de violencia desencadenados en Chile en octubre pasado poseían un distintivo carácter generacional por su composición etaria. En efecto, una encuesta (NUDESOG, 2020) realizada en las calles de Santiago entre el 8 y 29 de noviembre de 2019 a manifestantes mayores de 18 años participantes en las protestas, revela que la edad promedio de quienes hacían parte de la protesta era de 33 años, con una similar proporción entre mujeres y hombres, provenientes en su mayoría (49\%) de las comunas de Santiago Centro, Nuñoa, Puente Alto, Maipú, La Florida y Providencia, comunas todas de un segmento de clase baja no pobre, clase media baja, clase media intermedia y un segmento de clase alta. El nivel educacional declarado por los manifestantes llama la atención; en efecto, el 55\% declara haber completado la ES (32\% universitaria, 13\% técnico-profesional y un $10 \%$ posee un posgrado); un $9 \%$ tener ES; el $32 \%$ indica tener educación secundaria completa, y el 3\% dice poseer educación secundaria incompleta o un menor nivel de escolarización. De ser correctas estas cifras, resulta llamativa la alta proporción de personas con ES en todos sus niveles que participa en la protesta social, comparada con su prevalencia en la población entre 25 a 64 años que en Chile alcanza apenas a un $22 \%$, si bien en el tramo de edad de 25 a 34 aumenta hasta llegar al 30\%.

Adicionalmente, el $54 \%$ de los protestantes expresa ser el o la principal aportante al ingreso de su hogar; el $64 \%$ se identifica con posiciones políticas de izquierda; el 15\% señala ser de centro, $1 \%$ de derecha, $18 \%$ no identificarse con ninguna y $2 \%$ declara ser de otra posición o no responde. Se trata, por tanto, de un universo ideológico muy distinto al del conjunto de la población chilena, donde la mayoría no se identifica con ninguna posición política y quienes lo hacen se sitúan a sí mismos moderadamente a la izquierda o a la derecha del centro. Destaca además el alto porcentaje de manifestantes (93\%) que se encuentra 'muy insatisfecho' o 'insatisfecho' con la democracia en Chile, mientras sólo el 6\% se declara 'satisfecho' o 'muy satisfecho'.

Las principales demandas reveladas por los manifestantes se concentran en torno a pensiones $(75 \%)$, salud $(58 \%)$ y educación ( $57 \%$ ), seguidas por justicia social (23\%), una nueva Constitución (21\%), empleo y sueldos dignos $(16 \%)$, derechos humanos y no más impunidad (10\%). El 47\% afirma haberse manifestado en las calles de modo 'seguido' o 'muy seguido' durante los últimos 10 años; un 43\% 'a veces' o 'rara vez', y un 9\% 'nunca antes'. Sólo el 35\% menciona participar en una organización vinculada a la movilización, tales como centros estudiantiles, juntas de vecinos, partidos políticos u otras.

Por su lado, una encuesta del Centro de Estudios Públicos (CEP, 2020) respecto a la percepción de la protesta social y las expresiones de violencia aplicada entre el 28 de noviembre de 2019 y el 6 de enero de 2020 a personas mayores de 18 años de edad en todo el territorio nacional, arrojó, en términos gruesos, un grado relativo de aprobación hacia las protestas y un rechazo ambiguo de la violencia. Sobre las marchas comenzadas en octubre de 2019, el 55\% las aprueba, 11\% las rechaza, 10\% las apoyó inicialmente y luego las objetó, 7\% las rechazó inicialmente y después las respaldó, 15\% no las avaló ni desestimó, y $2 \%$ no sabe o no contesta. Las personas estiman que las razones fundamentales tras las manifestaciones son: la desigualdad (38\%), las bajas pensiones (16\%), el alto costo de la vida (16\%), la mala calidad de la salud y la educación públicas (13\%). En relación con diversas formas de protesta, aprueban 'siempre' o 'casi siempre' participar en una marcha (55\%); evadir el pago del transporte público cuando sube su 
precio $(25 \%)$ y participar en barricadas o destrozos $(8 \%)$. Sin embargo, esta última alternativa se justifica 'siempre' o 'casi siempre' según un 15\% de las personas entre 18 y 24 años de edad. Asimismo, en el segmento con mayor nivel educacional (12 años y más de escolarización), el 25\% la justifica (12\% 'siempre' y $13 \%$ 'a veces'), evidenciando un grado importante de radicalización en la población más joven y con mayor escolarización.

En otro ámbito de asuntos, el 56\% de la población cree que una nueva Constitución 'probablemente ayude a resolver los problemas', $6 \%$ considera que 'probablemente empeore la situación actual', 25\% opina que 'probablemente deje las cosas igual', en tanto que $13 \%$ no sabe o no contesta. El $64 \%$ de los encuestados piensa que 'la democracia es preferible a cualquier otra forma de gobierno', si bien sólo $6 \%$ cree que en Chile funciona 'bien' o 'muy bien', $44 \%$ cree que funciona 'regular'; $47 \%$ que funciona 'mal' o 'muy mal', y $4 \%$ no sabe o no responde.

Por último, una encuesta administrada entre el 6 y el 15 de enero de 2020 a mayores de 18 años de ambos sexos (FIEL y MORI, 2020) mostró que el $62 \%$ de las personas cree que 'el estallido social es consecuencia de la crisis económica', frente al 34\% que estima que 'la crisis económica es producto del estallido social'. El 93\% sostiene que la distribución del ingreso en Chile es injusta y $84 \%$ que en Chile no se respetan las leyes; $80 \%$ juzga que 'uno nunca es lo suficientemente cuidadoso en el trato con los demás' y sólo 18\% declara que 'se puede confiar en la mayoría de las personas'; $79 \%$ se muestra de acuerdo con las protestas, $69 \%$ opina que no se ha dado respuesta ninguna a las demandas sociales del estallido social; $56 \%$ dice pertenecer a la clase baja, $39 \%$ a la media y $5 \%$ a la clase alta, en contraste con tres años antes del estallido cuando el 19\% se identificaba como de clase baja, 66\% como de clase media y 16\% como de clase alta (PNUD, 2017: 164), lo que representa un vuelco significativo respecto de la autodefinición subjetiva de pertenencia de clase de la población.

\section{Elementos para una interpretación}

Durante el último cuarto de siglo el número de estudiantes de la ES prácticamente se quintuplicó en Chile, ampliándose el acceso hacia los grupos de ingresos medios y bajos. En efecto, la tasa bruta de cobertura aumentó durante el periodo desde 21.2\% en 1991 a 88.5\% en 2018 (World Bank Group, 2020). A su vez, conforme a información proporcionada por el Programa de las Naciones Unidas para el Desarrollo (PNUD, 2017: 96), la participación de los dos quintiles inferiores aumentó de $5 \%$ y $7 \%$ a $42 \%$ y $50 \%$, respectivamente; la del quintil tres de $13 \%$ a $50 \%$, y la de los dos quintiles superiores de $25 \%$ y $50 \%$ a $60 \%$ y $77 \%$, respectivamente. Entonces, ¿cómo explicar que hayan sido mayormente adultos jóvenes con ES, y estudiantes de este nivel, quienes se movilizaron masivamente en las calles a partir de octubre pasado?

Un factor determinante tiene que ver con el hecho de que "hay diferencias significativas en el tipo de instituciones. Los jóvenes del estrato alto estudian preferentemente en universidades de mejor calidad (medida por el número de años de acreditación), mientras que aquellos de estrato más bajo lo hacen en institutos profesionales y centros de formación técnica, así como en universidades de peor calidad" (PNUD, 2017: 305). En efecto, a las universidades de mayor calidad — según especifica el mismo estudio (PNUD, 2017: 97) - asiste el 9.2\% de los estudiantes del estrato social bajo, $17.5 \%$ del medio bajo, $31.5 \%$ del medio, y $66.2 \%$ del estrato alto. A su turno, los jóvenes del estrato bajo asisten mayoritariamente $(60.4 \%)$ a instituciones consideradas de menor calidad, lo cual significa que la ES no solo refleja, sino que también reproduce, la desigualdad social a través de una desigual distribución de las oportunidades de estudio (Leihy y Salazar, 2017).

Por otro lado, la extensa democratización del acceso experimentada por la ES chilena conlleva tres elementos adicionales que seguramente impactan en su vinculación con la protesta. Primero, las altas 
expectativas de los estudiantes que ingresan cada año, los cuales, en su mayoría, son primera generación dentro de sus familias que cursan estudios superiores. Así, el número de egresados de educación terciaria pasó desde 14900 en 1991 a 235705 en 2017 (World Bank Group, 2020). En general ellos suponen, al acceder a la educación superior, que con esto aseguran un significativo retorno económico, bajo la forma de un premio salarial al título profesional o técnico, junto con incrementar sus posibilidades de movilidad social ascendente, adquirir un status típico de clase media y asegurar los beneficios correspondientes a ese estrato.

Segundo, tales aspiraciones, formadas tempranamente durante la enseñanza primaria y secundaria, aparecen en Chile como desmesuradas. La prueba PISA (Programme for International Student Assessment) de 2015 preguntó a los jóvenes de 15 años acerca de cuál era el nivel escolar que esperaban alcanzar y Chile, después de Corea y Estados Unidos y junto con Canadá, ocupó el tercer lugar de más altas expectativas entre los países de la Organización para la Cooperación y el Desarrollo Económico (OCDE); efectivamente, un $66.6 \%$ y un $13.3 \%$, respectivamente, creen que completarán estudios profesionales o estudios técnicos. Para la OCDE (2017: 105), las cifras promedio correspondientes son $44 \%$ y $15 \%$. Igualmente, la prueba internacional PISA de 2013, al consultar a estudiantes de 15 años (que por tanto hoy se aprontan a ingresar al mercado laboral) sobre cuáles eran sus expectativas respecto a ocupar posiciones directivas de alto status ${ }^{5}$ en el futuro, constató que en Chile un $67 \%$ responde positivamente, situándose junto a México, Turquía e Israel entre los cuatro países con mayores expectativas de alcanzar dicho status (OECD, 2013). Resultados similares, aunque todavía más optimistas en tres puntos porcentuales, se habían obtenido antes en el caso de Chile sobre la base de PISA 2006 (OECD, 2012: 81) para jóvenes que hoy en su mayoría se encuentran incorporados al mercado de trabajo.

Tercero, sin embargo, y a contramano con tales expectativas, ya a fines de la primera década del presente siglo aparecen los primeros estudios que indican un mayor reconocimiento del riesgo de que tales aspiraciones podrían verse pronto frustradas. Se decía que el rápido crecimiento de profesionales y técnicos superiores podía provocar una suerte de saturación del mercado laboral de algunas profesiones, fenómeno que llevaría a una polarización salarial pudiendo además crear una inconsistencia de status y una pérdida de autonomía profesional para los graduados situados en la parte inferior de la pirámide de las respectivas profesiones (Brunner y Uribe, 2007: 232-236). Por su lado, estudios publicados por algunos economistas estimaban que, para un porcentaje de alumnos de ES, especialmente entre aquellos matriculados en instituciones de menor calidad (prestigio), existía la probabilidad de que, una vez ingresados al mercado laboral, la tasa privada de retorno de su inversión en capital humano avanzado fuese negativa, dejándolos en mal pie para saldar la deuda contraída para costear sus estudios (crédito estudiantil) (Iturrieta, 2018).

Investigaciones recientes subrayan cómo el proceso de democratización del acceso a la educación superior, y el consiguiente aumento del número de graduados en todo tipo de programas y carreras, han ido de la mano de una disminución de la rentabilidad de los títulos y grados profesionales, aunque de manera desigual entre éstos y presentando variaciones en función del origen socioeconómico y trayectorias educativas de los egresados (Klapp y Candía, 2016; Orellana, 2018). El estudio de Riquelme Silva

\footnotetext{
${ }^{5}$ Se refiere a ocupaciones de los grupos 1 y 2 dentro de la Clasificación Internacional Uniforme de Ocupaciones (CIUO). El grupo 1 comprende a legisladores, altos funcionarios y gerentes, y el grupo 2 a profesionales de las ciencias y de la ingeniería, la salud, la enseñanza, especialistas en organización de la administración pública y de empresas, profesionales de tecnología de la información y las comunicaciones, y profesionales en derecho, en ciencias sociales y culturales.
} 
y Olivares Faúndez (2015) señala, por ejemplo, que la rentabilidad de la enseñanza superior comienza a decrecer ya desde 2000 , cuando alcanzaba un $27 \%$, reduciéndose a $23 \%$ en 2009 y 19\% en 2011. En una dirección similar, Carrillo, Espinoza y Valenzuela (2018) indican que el retorno de la educación superior (universitaria y técnica) se ha reducido desde $228 \%$ a $157 \%$ de los ingresos de una persona sin educación entre 2000 y $2015 .{ }^{6}$ Adicionalmente, como se aprecia en la tabla 1, la tasa de desocupación en la población con estudios de educación superior se ha mantenido relativamente constante durante la última década, especialmente en el segmento que tiene entre 15 y 21 años de escolaridad, pero ha experimentado un significativo incremento durante el último año, en coincidencia con el fenómeno de la protesta social, seguramente agravado ahora por los efectos del Covid-19.

\section{Tabla 1. Tasa de desocupación de la población con 13 o más años de estudio}

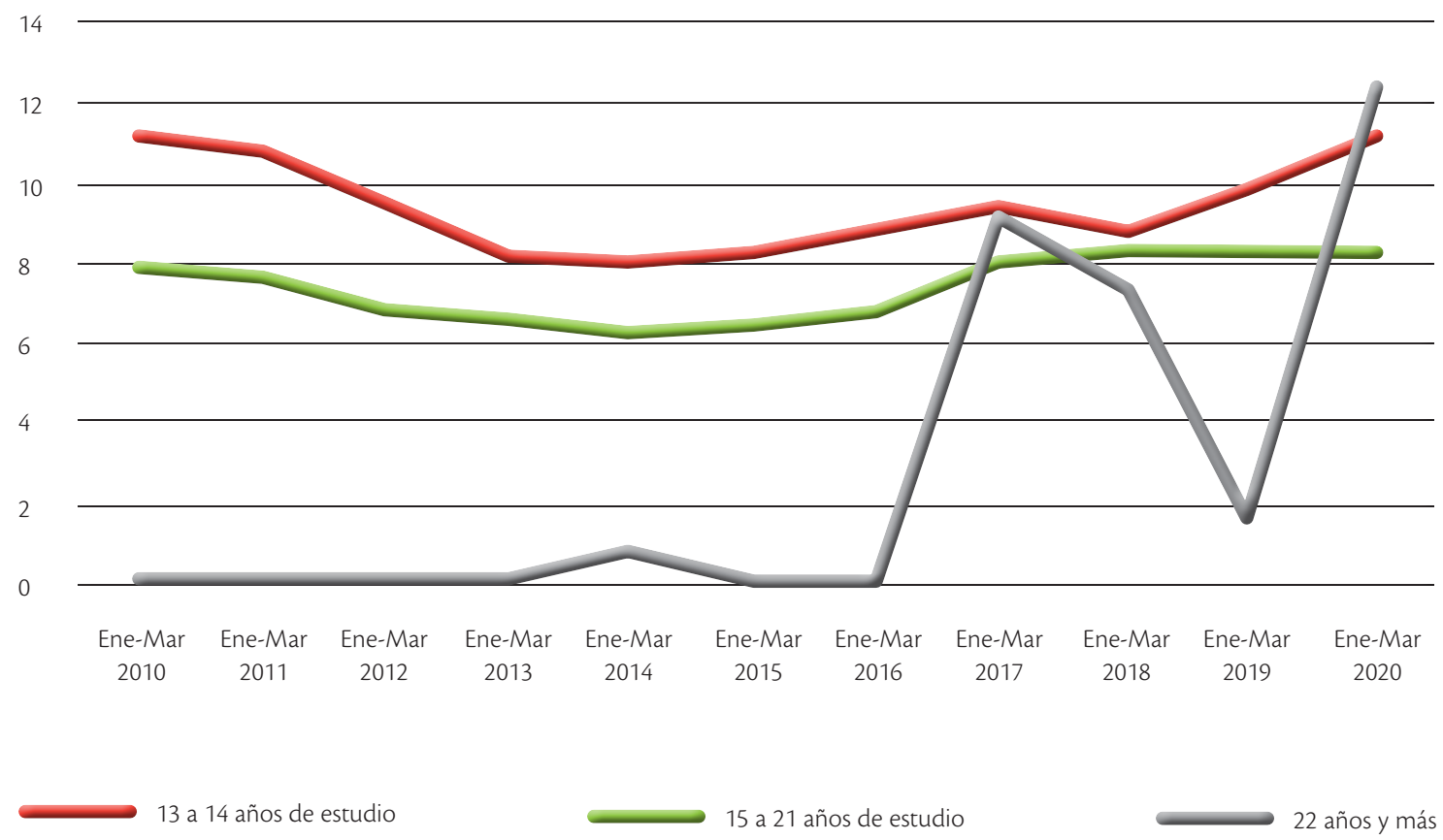


Bourdieu (2016) ha advertido que los procesos de masificación del acceso a certificados educacionales son ambiguos pudiendo, al final, volverse contra su propio propósito. En la sección titulada "una generación engañada" de su libro La Distinción, este autor se refiere al desajuste estructural entre aspiraciones y resultados que produce el sistema educacional en una fase de rápido crecimiento y de inflación de diplomas, cuando las oportunidades laborales ya no aseguran el retorno esperado. Esa desilusión colectiva dice, "se encuentra en la base de la desafección con respecto al trabajo y de las manifestaciones del rechazo de la finitud social, que está en la raíz de todas las fugas y de todos los rechazos constitutivos de la 'contra cultura' adolescente". Refiriéndose a la generación de estudiantes franceses de 1968, donde observa en plena acción ese "efecto de histéresis" como lo llama —o sea, desajuste entre aspiraciones y satisfacciones producido por que aquellas anteceden a éstas que pierden valor al masificarse- - observa el fenómeno del surgimiento de una "generación engañada y desengañada". Así:

La descualificación estructural que afecta al conjunto de los miembros de esta generación, destinados a obtener de sus titulaciones menos de lo que hubiera obtenido de ellas la generación precedente, se encuentra en la base de una especie de desilusión colectiva que lleva a esta generación engañada y desengañada a hacer extensiva a todas las instituciones la rebeldía unida al resentimiento que le inspira el sistema escolar (Bourdieu, 2016: 145).

Un fenómeno similar de divergencia entre expectativas y satisfacciones ocurre al momento en que muchos de estos jóvenes acceden al mercado del trabajo e inician una precaria vida profesional o, más bien, semi-profesional, en ocasiones cargando, además, con una deuda (Pérez-Roa y Ayala, 2019). En Chile, un grupo amplio de alrededor de 250000 jóvenes profesionales y técnicos ingresan cada año al mercado laboral bajo el efecto bourdieuano de la histéresis. En tal sentido, una joven kinesióloga chilena citada en un estudio a propósito de la generación desengañada, manifiesta: "[...] te 'dai' cuenta que tener el título, el cartón en la mano, uno dice, oh, ya, soy profesional, no significa nada, no te asegura que todos tus sueños, todas tus ideas queden como completas" (Ramos Matus, 2018), pasaje donde aparece claramente la discordancia entre el sueño y la realidad, entre las condiciones imaginadas del mercado laboral y sus condiciones actuales, entre el prestigio proclamado por, o atribuido a, la institución otorgante del título y el prestigio efectivo que sus diplomas comandan en el mundo ocupacional, entre el status e ingresos anticipados y aquellos reconocidos socialmente. Desde otro ángulo, un arquitecto que ha completado ya una parte importante de su vida profesional, en entrevista realizada para una tesis sobre representaciones de la movilidad social, expresa:

Yo lo que veo es que, por ejemplo, cuando yo me titulé de arquitecto, el arquitecto tenía un estatus en la sociedad que era mucho mayor del que tiene ahora. O sea, antes ser arquitecto era un prestigio en sí, porque los arquitectos sólo por serlo, se les abrían oportunidades y podían acceder a un estatus de vida que era destacado dentro de la sociedad, pero hoy, no te entrega un estatus en sí mismo (González-Madariaga, 2017).

Por su lado, un egresado de derecho de una universidad privada regional señala: "Hoy en día sacan y sacan y sacan profesionales, y uno encuentra muchísimo cesante ilustrado; gente con mucho título universitario, muchos estudios, pero que no consigue trabajo; está trabajando en ventas o en cualquier cosa para poder sobrevivir, porque al final lo que necesita es dinero 'pa' vivir" (Vásquez-Palma, 2017).

Con todo, la "generación desengañada" — si bien es un factor que juega un papel seguramente decisivo en el desencadenamiento de la protesta social - no 
opera como un factor aislado. Intervienen además otros factores, de mayor magnitud, que resultan de las transformaciones experimentadas por la sociedad chilena durante los últimos 30 años, y que afectan significativamente a dicha generación y al conjunto de la sociedad, tales como el impacto desestabilizador de la intensa modernización capitalista, la irrupción de una generalizada mercantilización de la vida, una individuación y contractualización de las relaciones humanas, la disolución de las comunidades tradicionales (Brunner, 1998, 2005) y la emergencia de una imprecisamente denominada "sociedad de clases medias" (Ariztía, 2016; Mac-Clure, 2012; Méndez, 2008). En efecto, se trata del surgimiento de una nueva estratificación de la sociedad, caracterizada por un fuerte aumento del número de profesionales, semi-profesionales y técnicos, trabajadores de cuello y corbata, personal de servicios calificados en el sector público y privado, personas con formación superior completa e incompleta que trabajan por su cuenta y un vasto segmento que habiendo superado la línea de pobreza y adquirido alguna credencial educacional, sin embargo apenas logran mantenerse por encima de ese umbral.

En juego hay pues aquí una definición amplia de clase media, cuya estratificación interna distingue usualmente entre estratos de clase media baja, intermedia y alta, dependiendo de ocupaciones, ingresos, niveles de consumo, posesiones y estilos de vida. En Chile, alrededor de un 70\% de la población se autocalifica como de clase media (Méndez, 2008), pudiendo definirse a ésta - para efectos de nuestro estudiocomo aquella compuestas por la clase baja no pobre (1.8 líneas de pobreza), la clase media baja (hasta tres líneas de pobreza) y la clase media intermedia (de 3 a 6 líneas de pobreza) que, en conjunto, suman el 64\% de la población mayor de 25 años (PNUD, 2017: 68-69). En general, las distintas estadísticas consultadas coinciden en que alrededor de 2017, en torno a la mitad de la población mayor de 25 años se ubicaba en los estratos bajos no pobres y los estratos medio-bajos que "presentan altos riesgos de caer en situación de pobreza ante episodios de desempleo o precarización del trabajo, enfermedades catastróficas, accidentes o desastres naturales" (CEPAL, 2019: 62).

Es probable que en dicho universo en riesgo, especialmente en el grupo de edad de 40 años o menos - de hecho, un 75\% de los protestantes encuestados después del estallido de octubre pasado tenía, como hemos señalado, 39 años o menos (Núcleo de Sociología Contingente (NUEDESOC), 2020)-, se encuentre el centro de gravedad de la protesta social, al igual que la explicación de su carácter multitudinario y la diversidad de sus demandas. Podría explicar además que una parte de las personas que declaran pertenecer a la clase media tenga dificultad para asumir esa pertenencia, debido al hecho de que con frecuencia ocupa posiciones poco definidas, en el límite entre la clase baja no pobre y la clase media baja. Más bien, se manifiesta allí una constante duda respecto de la ubicación personal y del grupo de referencia dentro de la jerarquía social y si podrán mantener esa posición, ascender a partir de ella o caer debajo de la línea de pobreza. Como señala una joven psicóloga egresada de una universidad privada no-selectiva, manifestando el riesgo siempre presente de descender: "No sé en qué clase social, no sé, me veo en un punto de desarrollo continuo; pero sí de que estaba abajo y ahora estoy más arriba, cuánto más arriba no sé, no sé si voy a tener más techo o voy a seguir, no sé si me voy a mantener, cachay" (Ramos Matus, 2018).

Todo indica, entonces, que la protesta social chilena fue, básicamente, una protesta de clase media en el sentido de esos tres grupos caracterizados previamente - bajo no pobres, medio bajo e incluso algunas fracciones no consolidadas del estrato medio intermedio y sus hijos y nietos-, siendo uno de los componentes más activos aquel conformado por jóvenes profesionales y técnicos que dentro de sus familias son la primera generación que cursó estudios superiores, estudiantes secundarios y de 
educación terciaria, y miembros inseguros de su posición dentro del estrato medio intermedio, muchos de ellos afectados por deudas (de consumo, estudio e hipotecaria), por la ralentización de la economía, la dificultad de encontrar empleo, los ingresos insuficientes y la precaria calidad de los servicios de salud, educación y previsión a los que acceden ellos y sus familias. Como señala en una reciente entrevista un analista de las izquierdas: "hay un pueblo nuevo, que es el que construyó el neoliberalismo, que no es el pueblo del siglo XX. Es el pueblo de estos profesionales que no son clase media, que les vendieron un cuento que no es" (Ruiz, 2020b); es decir, ese amplio y variado grupo de engañados y desengañados por el efecto de la histéresis. Si se deja de lado el curioso supuesto de que las clases o estratos nacen de las ideologías y que la experiencia de vida de los grupos emergentes es meramente reflejo de una falsa conciencia, la descripción del analista citado acierta en detectar que la sociedad chilena ha mudado y con ella, también, la base social de la protesta.

De manera que cabe plantear la hipótesis de que el fenómeno desencadenado por la inflación de credenciales y el efecto de histéresis reverberó tan intensamente en la sociedad chilena porque se imbricó con los efectos generados por las transformaciones experimentadas por aquélla durante los últimos 30 años. ${ }^{7}$ Es el resultado de la abrupta masificación de la educación superior coligada que se halla en la base del surgimiento de una sociedad de estratos medios, los que pugnan por alcanzar y consolidar posiciones más estables y justas dentro de la distribución de oportunidades y beneficios en un Estado que proclama, pero no garantiza, un número creciente de derechos sociales (Atria, 2018; Ruiz y Caviedes, 2020).

\section{Rol de la universidad ante a la protesta}

¿Cuál fue el rol de la educación superior - las universidades en particular - ante la protesta social y la crisis de gobernabilidad, que ahora último se ha ahondado además con los efectos de la pandemia del Covid-19? Para responder a esta pregunta desde el plano sociológico podemos recurrir a la tradición de análisis que en América Latina ha discutido la cuestión de la responsabilidad de la universidad frente a las crisis de la sociedad y su superación.

Tal cuestión ocupa un lugar central en el pensamiento latinoamericano, cuyo locus clásico se halla en los escritos sobre educación de José Medina Echavarría. Si la universidad había tendido a ser la institucionalización de la inteligencia, sostiene él, entonces su misión en situaciones de crisis debe ser orientar, y comprometerse con, la búsqueda de salidas que permitan superarla (Graciarena, 1980). Mas esto podía hacerse de diferentes maneras, que el autor identifica con tres modelos o tipos ideales de universidad: la universidad "torre de marfil", en la tradición de Guillermo von Humboldt; la universidad "militante", como la llamó, que se deja seducir por los ruidos de la calle, decía él; y la universidad "partícipe", desde su propia racionalidad científica, en los procesos de desarrollo nacional (Medina Echavarría, 1967).

Nuestro autor abogaba por una universidad 'partícipe' - que se hace parte de su sociedad y contribuye a la solución de sus problemas y crisis desde la razón científico-técnica - sin dejarse llevar ni hacia la 'universidad enclaustrada', tipo torre de marfil, ni hacia la 'universidad militante', comprometida a fondo con la acción política. Las universidades chilenas reaccionaron frente a la protesta social, y siguen haciéndolo hasta hoy bajo la pandemia, gruesamente con una mezcla de elementos provenientes de los tres modelos.

\footnotetext{
${ }^{7}$ Una hipótesis similar se ha propuesto para varios países latinoamericanos (Chiroleu y Marquina, 2017). Para un enfoque económico del fenómeno, véase Urzúa (2017).
} 
En general, primó en ellas la voluntad de volverse partícipes de la superación de las crisis que sufre la sociedad, recurriendo a su propia racionalidad. Esto es, mantener hasta donde fuera posible la continuidad de las labores de enseñanza o mitigar los efectos de su interrupción; poner la investigación - especialmente de las ciencias sociales y humanidades - al servicio de describir, entender, explicar e interpretar los procesos sociales en curso, primero los propios de la protesta y, enseguida, aquellos surgidos en reacción a la crisis sanitaria provocada por la pandemia; y, tercero, reforzar sus labores de vinculación con el entorno, intensificando los contactos con el entorno, la reflexión crítica y el razonamiento públicos sobre los problemas de la sociedad y dando la mayor proyección que las circunstancias permitían a las actividades culturales de cara a los públicos externos. Hay múltiples registros, crónicas, catastros y ensayos que dan testimonio del cumplimiento de este papel que Jaspers (1959) representó en su momento como el de la universidad que se convierte en la conciencia más lúcida de su época, junto con mantener activo el de su propia racionalidad. En términos de Medina: universidad partícipe es "aquella que enfrenta los problemas del día aceptándolos como tema riguroso de consideración científica, para afirmar únicamente lo que desde esa perspectiva se puede decir [...] Nada de su tiempo puede serle ajeno, pero sólo en la medida que pueda situarlo a la distancia que exige su búsqueda permanente de la verdad" (Medina Echavarría, 1967: 168-169). Este tipo ideal, como pudimos ver en Chile en este tiempo, nunca se expresa de una manera tan pura en la realidad, sino que presenta un carácter hibrido, con elementos de los otros dos modelos, como se verá a continuación.

Efectivamente, junto a esa función "partícipe", hubo además - en diferentes grados y con características diversas según cada caso- elementos propios de la institución "militante" en la mayoría de las universidades chilenas; es decir, aquella que se deja "invadir sin tamiz alguno por los ruidos de la calle y reproduce en su seno, en exacto microcosmos, todos los conflictos y pasiones de su mundo. La tarea científica desaparece y sólo se escuchan gritos en vez de razones" (Medina Echavarría, 1967: 169). Sin duda, a lo largo de este tiempo de protestas y pandemia hubo momentos de esa naturaleza, no al punto de transformar a las universidades en trincheras y barricadas - aunque también puntualmente eso ocurrió en ciertas instituciones-, sino como fenómenos emergentes en medio de la agitación social, transmitidas por la experiencia que estaban viviendo los jóvenes, por la propia vocación "partícipe" de los profesores y por el papel estratégico que, en cualquier caso, juegan las instituciones universitarias en las democracias contemporáneas.

Por fin, con la pandemia y el confinamiento de la población en general, y de los estudiantes y profesores en particular, las universidades han debido mudar nuevamente y convertirse, rápida y forzosamente, en plataformas de educación a distancia. La vibrante comunidad presencial, interactiva, gregaria, dio paso a instituciones virtuales, con sus miembros puestos en cuarentena, donde primó aquello que Medina ya casi no creía posible - 'Si la 'universidad enclaustrada' ha sido siempre excepcional y hoy casi imposible, 'torre de marfil' tan sólo en el denuesto", escribía él (Medina Echavarría, 1967: 168)-, es decir, unas instituciones del distanciamiento físico, sin acercamiento interpersonal, sólo con intercambios virtuales. Pero, además, una institución que, en la experiencia de los profesores, es auténticamente una 'universidad enclaustrada', con algo de clima monástico, cada cual encerrado en su hogar, entre sus libros; y que, en la experiencia de los estudiantes, resulta frecuentemente una vivencia artificial, frustrante, desagregada, sin estructura ni las rutinas ordinarias que dan orden al caos de la vida.

He ahí pues el difícil balance que las universidades chilenas han intentado establecer en estos tiempos de protesta y pandemia, conjugando los elementos propios de tres tipos ideales de institución que recorren el imaginario académico latinoamericano del último medio siglo. 
En el trasfondo de estos procesos subsiste el hecho de que la universidad es una parte fundamental de las transformaciones que experimenta nuestra sociedad.

Lo es de un lado, como se vio, por la inevitable popularización de las credenciales profesionales y técnicas que trae consigo la expansión de la ES, desencadenando un doble efecto de histéresis en relación con las expectativas de la vida estudiantil y de la vida laboral; del otro, por la presión del entorno que fuerza a abandonar la reflexividad racional propia del campo académico y las disciplinas para volcarse a la calle, sustituyendo aquella racionalidad por el calor de las trincheras; en breve, cambiando la serenidad por el fervor. O bien, imponiendo un inevitable "enclaustramiento" donde vuelve a brillar, por un instante, la promesa de "libertad y soledad" que en el nacimiento de la moderna universidad alemana se estimó era la base de la auténtica cultura académica (Nybom, 2003). El principal contraste que surge en tales circunstancias es entre la universidad "militante" y alguna versión contemporánea de la "torre de marfil".

Según señala un promotor del diseño militante, "lo que está en debate, es la creación de un vínculo político orgánico entre la universidad y la sociedad, que ponga fin al aislamiento de la universidad", de modo tal de ser más receptiva ante las demandas sociales de grupos que carecen de poder para imponerlas por sí mismos (Santos, 2007: 84). Dicho directamente, una universidad que se propone desempeñar un papel estratégico y de vanguardia en la transformación social y, en lo posible, en la revolución y liberación nacional.

Este componente de compromiso político, ideológico y de lucha social está en el núcleo de una tradición latinoamericana del ideal de universidad, elaborada ya bien desde la perspectiva de los movimientos estudiantiles (Donoso, 2017) o bien por las narrativas de la reforma universitaria que arrancan desde Córdoba en 1918 (Sader, Aboites y Gentili,
2008). El ideal de universidad de Medina Echevarría corre en un sentido distinto, contrario incluso. Según él, en efecto, la responsabilidad de la inteligencia - título de uno de sus libros (Medina Echavarría, 1943) - consiste, especialmente en tiempo de protestas y crisis, en un permanente "compromiso con las alternativas de salida", buscadas - diríamos hoydesde el propio ethos comunicativo que define a la universidad (Habermas, 1987).

En cambio, la dialéctica entre universidad "militante" y "enclaustramiento" universitario encuentra su punto de equilibrio - por necesidad precario e inestable - justamente en el tercer tipo ideal de Medina Echavarría, la universidad "partícipe", que construye su identidad a partir de la racionalidad científico-técnica y reflexiva propia del cultivo de las disciplinas. Esta forma de universidad, constantemente interpelada desde ambos polos, de la protesta movilizada en las calles y del retraimiento monástico, es la que se ha encarnado socialmente durante los últimos meses en Chile, dentro de un entorno de crisis y resistencia. Así ha buscado perseverar en su misión formativa, de investigación reflexiva y de contribución a la deliberación pública, asumiendo el rol intelectual que le cabe en la sociedad.

\section{Conclusiones}

La tesis interpretativa planteada en el presente ensayo sostiene que la ES chilena ha cambiado su rol en el seno de una sociedad en crisis, incorporando una combinación de elementos provenientes de tres modelos: del confinamiento, la militancia y el compromiso de participación sobre la base de su propia racionalidad institucional. A lo largo del siglo XX, el movimiento estudiantil sostuvo que el acceso a la ES debía ser para todos y la universidad comprometerse con el cambio de la sociedad y sus relaciones de poder. Al contrario, sectores de la academia y la sociedad han afirmado que la universidad debía tomar distancia de los conflictos sociopolíticos y situarse por encima de las querellas entre diferentes grupos 
de poder. En la actualidad, dichos planteamientos se han visto modificados por la doble crisis de las protestas y la pandemia. No sólo se ha universalizado la ES, aunque con accesos diferenciados por capital económico y capital cultural de los estudiantes, sino que las universidades han asumido, además, un papel más relevante en sociedades cuyas economías se integran desde la periferia al sistema del mundo del capitalismo global (Labraña, Brunner y Álvarez, 2019). La propia sociedad chilena ha modificado su estructura de clases y estratos a partir de esa masificación y universalización de la educación superior, creando nuevos sujetos que, mediante la protesta social, buscan una mejor distribución de bienes materiales y simbólicos, beneficios y oportunidades, propios de un moderno Estado benefactor.

Tras esa renovada lógica de protesta se encuentra el efecto bourdieuano de histéresis que crea unas generaciones de profesionales y técnicos que no sólo se sienten "desengañados" respecto de las promesas de movilidad social y de participación en las oportunidades de vida que creían aseguradas por los estudios superiores sino, además, se sienten "engañados" por un sistema y sus élites políticas y empresariales que, en la práctica, no estarían en condiciones de garantizar esas promesas. De este modo la desilusión colectiva de esas generaciones se extiende al conjunto de la institucionalidad político-económica y cultural del orden establecido - capitalismo periférico integrado al mundo global y democracia representativa - adquiriendo un carácter potencialmente anti-sistémico. A ello se agrega durante los últimos meses la crisis sanitaria causada por el Covid-19 y la crisis social proveniente del coma inducido de la economía y del confinamiento generalizado de la población. Doblemente remecidas, las universidades deben hacer frente a su propia crisis de identidad y renovar el balance del tipo de comunidad imaginada que desean ser. Para ello recurren, centralmente, a elementos de su propia racionalidad técnico-científica y de conciencia reflexiva junto con retomar a elementos de las tradiciones de la universidad "militante" y la universidad "torre de marfil".

Por cierto, como muestra este artículo, esa dinámica de renovación se da en un cuadro más general que contextualiza los efectos de histéresis postulados como mecanismo explicativo de la protesta social, tales como la carga onerosa de la deuda estudiantil que sobrellevan las generaciones de graduados recientes; la mercantilización generalizada de la vida (Peña, 2017); las precarias condiciones de acceso a la salud y la vivienda de los estratos bajos no pobres y de clase media; la sensación de la población de ser continuamente abusada por las relaciones clasistas de poder y de hallarse insatisfecha por la explosión generalizada de expectativas producidas por varias décadas de desarrollo económico-social ininterrumpido.

En este cuadro, el papel de la universidad cambia al transformarse en un elemento clave para la reproducción de los estratos bajos, vulnerables y precarios de la nueva clase media que se expande a partir del acceso universal a la educación superior y la multiplicación de las credenciales universitarias.

En el caso chileno, el régimen de economía política de la ES, así como su organización, financiamiento y gobernanza, asumen la forma de un capitalismo académico de alto privatismo (Brunner, Ganga y Rodríguez-Ponce, 2018), donde el sentido mismo de la universidad y su carácter de bien público se hallan en continua discusión (Guzmán-Valenzuela, Barnett y Labraña, 2020). Fuera de discusión está el hecho que la ES ha devenido el principal canal para participar en el mercado de certificados educativos, cuya dinámica resulta luego esencial para la distribución y legitimación de posiciones ocupacionales, premios salariales, status adquiridos y estilos de consumo y de vida. En medida creciente, entonces, la estructura social de clases y estratos medios de la sociedad chilena descansa sobre esa función mediadora y legitimadora de la ES y la ideología meritocrática y mesocrática que la acompaña, pero no la asegura. 
Puesta la ES frente a la protesta social que ella misma, estructuralmente, contribuye a generar, ¿qué rol asume la universidad frente a aquella? ¿Y cómo se comporta, adicionalmente, ante la pandemia que la obliga a confinarse y actuar a cierta distancia de la sociedad? Hemos respondido a estas interrogantes recuperado el pensamiento sociológico de José Medina Echavarría sobre la ES y sobre la función de las universidades en la crisis y ante los retos del desarrollo latinoamericano, que en el presente se han vuelto extraordinariamente complejos. Las opciones para las universidades son básicamente tres, cuya combinación en la práctica resulta en una diversidad de estrategias. Las universidades pueden replegarse sobre sí mismas y transformarse temporalmente en una espléndida torre de marfil, dedicada a la ciencia

\section{Agradecimientos}

Los autores agradecen el soporte del Proyecto FONDECyT No 1180746 - Sistema universitario chileno: gobernanza del capitalismo académico y calidad de las instituciones.

\section{Referencias}

Araujo, Kathya (ed.) (2020), Hilos tensados: para leer el octubre chileno, Santiago de Chile, Instituto de Estudios Avanzados (IDEA-USACH)/Editorial Usach.

Ariztía, Tomás (2016), "Clases medias y consumo: tres claves de lectura desde la sociología”, Polis. Revista Latinoamericana, vol. 14, núm. 43, pp. 435-459.

Atria, Fernando (2018), "La fracturada relación entre política y sociedad (o: sobre el sentido político de los movimientos sociales)", Revista Anales, núm. 14, pp. 203-216.

Bourdieu, Pierre (2016), La Distinción. Criterios y Bases Sociales del Gusto, Barcelona, Taurus-Penguin Random House.

Brunner, José Joaquín (2005), “Con ojos desapasionados. Ensayos sobre la cultura en el mercado", en Consejo Nacional de la Cultura, Chile: 15 años de cambio cultural, por la ciencia en el aislamiento de su propia libertad; o bien, pueden salirse de sí mismas para volcarse hacia la calle y ahí fundirse con la protesta social reclamando para sí un lugar en la lucha por transformar la sociedad; o bien, pueden comprometerse con el planeamiento del futuro y la reforma de la sociedad a partir de su propia racionalidad de valores científico-técnicos y de participación en el espacio deliberativo público. La experiencia de los últimos meses, desde octubre pasado hasta ahora, agosto de 2020, nos enseña que las universidades no funcionan con ninguno de esos modelos en su pureza de tipo ideal, sino que combinan elementos tomados de los tres y elaboran así sus estrategias que les permiten adaptarse al entorno y cumplir con su misión, aún en medio de condiciones adversas.

Santiago de Chile, Consejo Nacional de la Cultura, pp. 35-43.

Brunner, José Joaquín (1998), "Malestar en la sociedad chilena: ¿de qué, exactamente, estamos hablando?”, Estudios Públicos, núm. 72, pp. 173-198.

Brunner, José Joaquín y Francisco Ganga (2018), "Factores a considerar en la reforma de la educación superior: un análisis a partir del caso chileno", Opción: Revista de Ciencias Humanas y Sociales, núm. 86, pp. 49-70.

Brunner, José Joaquín y Francisco Ganga (2016), "Reflexiones en torno a economía política y gobernanza de los sistemas nacionales e instituciones de educación superior en América Latina”, Interciencia, vol. 41, núm. 8, pp. 573-579.

Brunner, José Joaquín y Julio Labraña (2018), "Financiamiento de la educación superior, gratuidad y proyecto de nuevo crédito estudiantil", Debates de Política Pública, núm. 31.

Brunner, José Joaquín y Daniel Uribe (2007), Mercados universitarios: el nuevo escenario de la educación superior, Santiago de Chile, Ediciones Universidad Diego Portales. 
Brunner, José Joaquín, Francisco Ganga y Emilio Rodríguez-Ponce (2018). "Gobernanza del capitalismo académico: aproximaciones desde Chile", Revista Venezolana de Gerencia, vol. 23, núm. 1, pp. 11-35.

Carrillo, Francisco, Sebastián Espinoza y Andrea Valenzuela (2018), Mercado laboral y educación en Chile: principales tendencias y resultados, Santiago de Chile, Comisión Nacional de Productividad.

Castiglioni, Rossana (2020), "La política chilena en tiempos de pandemia: entre la (des)movilización social y la crisis sanitaria”, Nueva Sociedad, núm. 287.

Centro de Estudios Públicos (CEP) (2020), Estudio Nacional de Opinión Pública $\mathcal{N}^{\circ}$ 84, diciembre 2019, Santiago de Chile, GEP.

Comisión Económica para América Latina y el Caribe (CEPAL) (2019), Panorama social de América Latina 2019, Santiago de Chile, CEPAL.

Contraloría General de la República (GGR) (2018a), Financiamiento fiscal a la educación superior, Santiago de Chile, GGR.

CGR (2018b), Financiamiento fiscal del acceso gratuito a las instituciones de educación superior - 2017, Santiago de Chile, GGR.

Chiroleu, Adriana y Monica Marquina (2017), "Democratisation or credentialism? Public policies of expansion of higher education in Latin America", Policy Reviews in Higher Education, vol. 1, núm. 2, pp. 139160.

Consejo Nacional de Educación (CNED) (2020), Indicadores por dimensión - Contexto institucional - Estudiantes - Plantel académico, Santiago de Chile, CNED.

Comisión Nacional de Investigación Científica y Tecnológica (CONICyT) (2020), Data ciencia. Dimensiones de la producción nacional, Santiago de Chile, CONICyT.

Disi Pavlic, Rodolfo (2020), "Policies, parties, and protests: explaining student protest events in Latin America", Social Movement Studies, vol. 19, núm. 2, pp. 183-200.

Disi Pavlic, Rodolfo (2018), "Sentenced to debt: explaining student mobilization in Chile", Latin American Research Review, vol. 53, núm. 3.

Donoso Romo, Andrés (2017), “Movimientos estudiantiles universitarios en la época contemporánea de América Latina: elementos para pensar un modelo de aproximación histórica”, en Renate Marsiske (ed.), Movimientos Estudiantiles en la Historia de América Latina (Volumen V), México, Instituto de Investigaciones sobre la Universidad y la Educación, UNAM, pp. 57-84.

El Economista América (2019), "Ocho datos que permiten dimensionar los efectos de las protestas en Chile", en El Economista América, 14 de noviembre, <https:// www.eleconomistaamerica.cl/economia-eAm-chile/ noticias / 10199287/11/19/-Ocho-datos-que-reflejanlos-efectos-de-las-protestas-en-Chile.html> (Consulta: mayo de 2020).

Fundación Instituto de Estudios Laborales (FIEL) y Market Opinion Research International (MORI) (2020), Barómetro del trabajo enero 2020, Santiago de Chile, FIEL/ MORI.

González López, Felipe (2018), "Crédito, deuda y gubernamentalidad financiera en Chile", Revista mexicana de sociología, vol. 80, núm. 4, pp. 881-908.

González Madariaga, Valentina (2017), "Trayectorias entre la autoafirmación y la frustración en la sociedad chilena. Representaciones de meritocraciay desigualdad en quienes han vivido movilidad social ascendente", Tesis para optar al grado de Magister en Ciencias Sociales, mención Sociología de la Modernización, Santiago de Chile, Facultad de Ciencias Sociales de la Universidad de Chile.

González, Ricardo y Carmen Le Foulon (2020), “The 2019-2020 Chilean protests: a first look at their causes and participants", International fournal of Sociology.

Graciarena, Jorge (1980), "Universidad, inteligencia e ideología. En torno a algunas ideas de José Medina Echavarría”, en Jorge Graciarena y otros, Universidady desarrollo en América Latina y el Caribe, Caracas, CRESALCUNESCO, pp. 9-28.

Guerra, Sergio (2015), "El retorno del carnaval. Politización del carnaval y carnavalización de la política en el movimiento estudiantil chileno del 2011", Tesis para optar al grado de licenciado en artes con mención en teoría e historia del arte, Santiago de Chile, Facultad 
de Artes de la Universidad de Chile.

Guzmán-Concha, Cesar (2012), "The students' rebellion in Chile: occupy protest or classic social movement?", Social movement studies, vol. 11, núm. 3-4, pp. 408-415.

Guzmán-Valenzuela, Carolina, Ronald Barnett y Julio Labraña (2020), "Consensus and dissensus: changing perceptions of the public dimension of universities in a marketised environment", fournal of Higher Education Policy and Management, vol. 42, núm. 1, pp. 49-66.

Habermas, Jurgen (1987), "The idea of the university: learning processes",New German Critique, núm. 41, pp. $3-22$.

Iturrieta, Sandra (2018), "Masificación, segmentación y fragmentación de la educación superior chilena: Bienestar subjetivo de profesionales", Revista de Ciencias Sociales, vol. XXIV, núm. 3, pp. 83-96.

Jaspers, Karl (1959), The idea of the university, Boston, Beacon Press.

Klapp, Francisca y Alejandra Candia (2016), "Estimación del premio o retorno a la educación en Chile", Libertad y Desarrollo. Serie Informe Social, núm. 162.

Labraña, Julio (2018), "La primavera chilena: ni conservadora ni revolucionaria. Una explicación sociológica del significado histórico del movimiento universitario chileno del año 2011", Revista Calidad en la Educación, vol. 48, pp. 251-272.

Labraña, Julio, José Joaquín Brunner y Javier Álvarez (2019), "Entre el centro cultural y la periferia organizacional: la educación superior en América Latina desde la teoría de sistemas-mundo de Wallerstein", Revista Estudios Públicos, núm. 156, pp. 107-141.

Leihy, Peodair y José Miguel Salazar (2017), "The moral dimension in Chilean higher education's expansion", Higher Education, vol. 74, núm. 1, pp. 147-161.

Mac-Clure, Oscar (2012), "Las nuevas clases medias en Chile: un análisis de cohortes", Revista CEPAL, núm. 108, pp. 169-182.

Martuccelli, Danilo (2020), "El largo octubre chileno: bitácora sociológica", en Kathya Araujo (ed.), Hilos tensados: para leer el octubre chileno, Santiago de Chile,
Instituto de Estudios Avanzados (IDEA-USACH)/ Editorial Usach, pp. 369-376.

Medina Echavarría, José (1967), Filosofia, educación y desarrollo, México, Siglo XXI Editores.

Medina Echavarria, José (1943), Responsabilidad de la inteligencia, México, Fondo de Cultura Económica.

Méndez, María Luisa (2008), "Middle class identities in a neoliberal age: tensions between contested authenticities", The Sociological Review, vol. 56, núm. 2, pp. 220-237.

Metro de Santiago (2019), Balance daños 19 de octubre 2019, Santiago de Chile, Metro de Santiago, octubre.

Ministerio del Interior y Seguridad Pública (2019), Decreto Núm. 472 - Santiago, 18 de octubre de 2019. Diario Oficial, Núm. 42.481-B, Santiago de Chile, Ministerio del Interior y Seguridad Pública.

Nybom, Thorsten (2003), "The Humboldt legacy: reflections on the past, present, and future of the European university", Higher Education Policy, vol. 16, núm. 2, pp. 141-159.

Noticias ONU (2019), "Los Carabineros y militares en Chile cometieron graves violaciones de derechos humanos", en Noticias ONU. Mirada global, historias humanas, 13 de diciembre, disponible en <https:// news.un.org/es/story/2019/12/1466641> Consulta: mayo de 2020).

Núcleo de Sociología Contingente (NUDESOC) (2020), Encuesta Zona Cero - Informe de Resultados, noviembre de 2019 - marzo de 2020, Santiago de Chile, NUDESOC.

Organisation for Economic Co-operation and Development (OECD) (2019), Education at a Glance 2019: OECD Indicators, París, OECD Publishing.

OECD (2017), PISA 2015 Results (Volume III): Students' WellBeing, París, OECD Publishing.

OECD (2013), "Career Aspirations of 15-Year-Olds", en Economic, Environmental and Social Statistics, París, OECD Publishing (OECD Factbook 2013).

OECD (2012), Education at a Glance 2012: OECD Indicators, París, OECD Publishing.

Orellana, Natalia (2018), "Consideraciones sobre empleabilidad en educación superior", Calidad en la 
educación, núm. 48, pp. 273-291.

Peña, Carlos (2020), Pensar el malestar. La crisis de octubre y la cuestion constitucional, Santiago de Chile, Editorial Taurus.

Peña, Carlos (2017), Lo que el dinero sí puede comprar, Santiago de Chile, Editorial Taurus.

Pérez-Roa, Lorena y M. Constanza Ayala (2019), "The transition to the adult world with debt: characterizations of new economic insecurities of indebted young professionals in Santiago de Chile", Journal of Touth Studies, vol. 23, núm. 5, pp. 1-19.

Programa de las Naciones Unidas para el Desarrollo (PNUD) (2017), Desiguales. Orígenes, cambios y desafios de la brecha social en Chile, Santiago de Chile, PNUD.

Ramos Matus, Camila (2018), "Decisiones educativas y valoración de la educación superior en la trayectoria académica de los profesionales primera generación universitaria", Memoria para optar al título profesional de Socióloga, Santiago de Chile, Facultad de Ciencias Sociales de la Universidad de Chile.

Riquelme Silva, Guillermo y Víctor Olivares-Faúndez (2015), "Rentabilidad del trabajo en Chile: análisis de la evolución de los retornos por nivel educativo", Ciencia \& Trabajo, vol. 17, núm. 52, pp. 69-76.

Ruiz, Carlos (2020a), Octubre chileno. La irrupción de un nuevo pueblo, Santiago de Chile, Editorial Taurus.

Ruiz, Carlos (2020b), "La izquierda chilena no tiene proyecto. Entrevista con Pablo Vergara", Revista Electrónica Guion Bajo disponible en: <https://www. guionb.com/entrevistas/carlos-ruiz-la-izquierdachilena-no-tiene-proyecto/>.

Ruiz, Carlos y Sebastián Caviedes (2020), "Estructura y conflicto social en la crisis del neoliberalismo avanzado chileno", Espacio Abierto, Cuaderno Venezolana de Sociología, vol. 29, núm. 1, pp. 86-101.
Sader, Emir, Hugo Aboites y Pablo Gentili (eds.) (2008), La Reforma Universitaria: desafios y perspectivas noventa años después, Buenos Aires, CLACSO.

Santos, Boaventura de Sousa (2007), La universidad en el siglo XXI. Para una reforma democrática y emancipatoria de la universidad, La Paz, Cides-Umsa/Asdi/Plural Editores.

Somma, Nicolás, Matías Bargsted, Rodolfo Disi Pavlic y Rodrigo Medel (2020), "No water in the oasis: the Chilean Spring of 2019-2020", en Social Movement Studies, DOI: 10.1080/14742837.2020.1727737.

Stange, Hans, Antoine Faure, Claudia Lagos, Claudio Salinas, René Jara y Alejandro Lagos (2019), Rabia. Miedos, abusos y desórdenes en el oasis chileno, Santiago de Chile, Luis Emilio Recabarren.

Tironi, Eugenio (2020), El Desborde. Vislumbres y aprendizajes del 18-O, Santiago de Chile, Editorial Planeta.

United Nations Educational, Scientific and Cultural Organization (UNESCO) (2020), Glossary, París, UNESCO.

UNESCO (2019), Gross enrolment ratio, tertiary education, París, The UNESCO Institute for Statistics, UNESCO.

Urzúa, Sergio (2017), "The economic impact of higher education”, en María Marta Ferreyra, Ciro Avitabile, Javier Botero Álvarez, Francisco Haimovich Paz y Sergio Urzúa, At a crossroads: Higher education in Latin America and the Caribbean, Washington, The World Bank, pp. 115-148.

World Bank Group (2020), Education statistics: tertiary education, Washington, World Bank.

Vásquez Palma, Óscar (2017), "Educación superior y movilidad social en universidades privadas de baja selectividad: el caso chileno", Tesis para optar al grado de Doctor en Ciencias Sociales, Santiago de Chile, Facultad de Ciencias Sociales de la Universidad de Chile.

\section{Cómo citar este artículo:}

Brunner, José-Joaquín, Francisco Ganga-Contreras y Julio Labraña-Vargas (2020), "Universidad y protesta social: una reflexión desde Chile", Revista Iberoamericana de Educación Superior (RIES), vol. XI, núm. 32, pp. 3-22, DoI: https://doi.org/10.22201/ iisue.20072872e.2020.32.814 [Consulta: fecha de última consulta]. 